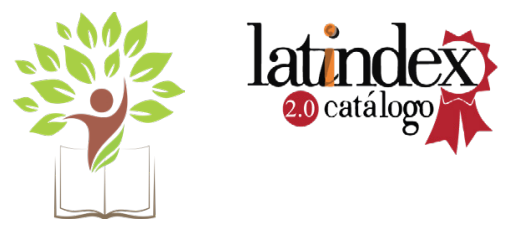

\title{
Posibilidades y dificultades de implementación de circuitos de proximidad comercial. Caso Asociación Valle de El Chaco: del productor al consumidor
} Possibilities and difficulties of implementation of commercial proximity circuits. The Chaco Valley Association case: from producer to consumer

Possibilidades e dificuldades de implantação de circuitos comerciais de proximidade. Caso da Associação Vale de El Chaco: do produtor ao consumidor

\begin{abstract}
Jorge Alejandro Batres Quevedo / Universidad Regional Amazónica Ikiam, Ecuador / jorge.batres@ikiam.edu.ec
\end{abstract}

Recibido: $16 / 7 / 2021$

Aceptado: 16/10/2021

Publicado: 4/11/2021

\section{RESUMEN}

La soberanía y la seguridad alimentarias de Ecuador dependen, en buena medida, de la producción de la agricultura familiar campesina; sector productivo que se ha visto afectado por la estructura agraria excluyente, el abandono del Estado, el despoblamiento rural y, más recientemente, la crisis sanitaria provocada por la covid-19. En este contexto, hace falta identificar acciones que permitan mejorar la situación actual de las asociaciones productivas. Esto será posible si se reduce la intermediación entre los productores y los consumidores, pues se bajarán los costos de comercialización, mejorarán los ingresos de los productores y se ofrecerán precios asequibles a los consumidores. Para valorar la factibilidad de esta posibilidad se analizó la oferta de productos agropecuarios de la Asociación Valle de El Chaco, así como la viabilidad de mejorar la forma de distribución aplicando circuitos de proximidad comercial. Con la información recolectada en entrevistas en línea y la observación directa, se diseñaron de forma participativa tres opciones de distribución que cumplían con los fines esperados. Sin embargo, se detectó que la eficiencia de dichas opciones dependía de las condiciones y capacidades de los productores, por lo cual se analizaron las debilidades, amenazas, fortalezas y oportunidades, y se identificaron las estrategias de acción.

Palabras clave: agricultura familiar campesina, asociacionismo, canasta de alimentos, comercialización, productos agropecuarios 


\begin{abstract}
Ecuador's food sovereignty and security depend, to a large extent, on the production of peasant family agriculture, a productive sector that has been affected by the exclusive agrarian structure, the abandonment of the State, rural depopulation and, more recently, the health crisis caused by covid-19. In this context, it is necessary to identify actions to improve the current situation of productive associations. This will be possible if the intermediation between producers and consumers is reduced, as marketing costs will be lowered, producers' incomes will improve, and consumers will be offered affordable prices. To assess the feasibility of this possibility, analyzed the supply of agricultural products of the Chaco Valley Association, as well as the feasibility of improving the distribution method by applying commercial proximity.
\end{abstract}

Keywords: agricultural products, associations, commercialization, food basket, peasant family farming

\title{
RESUMO
}

A soberania e a segurança alimentar do Equador dependem, em grande medida, da produção da agricultura familiar camponesa, setor produtivo que tem sido afetado pela estrutura agrária excludente, o abandono do Estado, o despovoamento rural e, mais recentemente, a crise sanitária provocada por covid-19. Nesse contexto, é necessário identificar ações para melhorar a situação atual do associativismo produtivo. Isso será possível se a intermediação entre produtores e consumidores for reduzida, pois, os custos de comercialização serão reduzidos, a renda dos produtores aumentará e os consumidores terão preços acessíveis. Para avaliar a viabilidade dessa possibilidade, analisou a oferta de produtos agrícolas da Associação Vale de El Chaco, bem como a viabilidade de melhorar o método de distribuição por meio da aplicação de circuitos comerciais de proximidade. Com as informações coletadas em entrevistas online e observação direta, foram traçadas três opções de distribuição de forma participativa que atenderam aos propósitos esperados. No entanto, detectou-se que a eficiência dessas opções dependia das condições e capacidades dos produtores, para os quais foram analisadas as fragilidades, ameaças, potencialidades e oportunidades, e identificadas estratégias de ação.

Palavras chave: agricultura familiar camponesa, associações, cesta básica, comercialização, produtos agrícolas

\section{INTRODUCCIÓN}

De acuerdo con Pino et al. (2018), Ecuador recibe un importante aporte económico de la producción agrícola, lo cual se expresa en cuatro indicadores obtenidos por la agricul- tura en los últimos 17 años: aportó el $8.4 \%$ del PIB total, contribuyó con el $34 \%$ de las divisas que necesita Ecuador para sostener la dolarización, generó empleo al $25 \%$ de la 
población económicamente activa y cubrió el $95 \%$ de la demanda interna de los alimentos que la población consume. En otras palabras, la producción agrícola de Ecuador tiene condiciones para garantizar tanto la soberanía como la seguridad alimentaria de su población; sin embargo, los autores señalan tres factores que amenazan este sector productivo: la migración del campo a la ciudad, el modelo agrícola excluyente y la falta de inversión en la agricultura por parte del Estado.

Según estimaciones de Villacís y Carrillo (2012), la relación entre la población urbana y la rural se invirtió entre 1950 y 2010 . En el primer año, la población rural representaba el $72 \%$ de la población total de Ecuador, mientras que en el segundo constituía apenas el $37 \%$. Es decir, en sesenta años la población rural de Ecuador se redujo cerca de la mitad, lo que representa un riesgo para la producción agrícola, pues el fenómeno está asociado al abandono de esta actividad por opciones más rentables en los centros urbanos. Lo anterior es lo que Srinivasan y Rodríguez (2016) nombran despoblamiento rural, y que, según ellos, produce un tránsito hacia economías no agrarias en las cuales la producción local de alimentos se ve afectada, generándose dependencia de su exportación para satisfacer la demanda.

Entre otras cuestiones, se reconoce que el despoblamiento rural se debe a la concentración de la tierra y a la reducción de la mano de obra por integración de tecnologías en la producción agrícola, pero, sobre todo, a las dificultades para acceder a la tierra. Según Sarandón (2020), en Latinoamérica persiste un modelo agrícola excluyente y tradicional, caracterizado por una estructura de propiedad del suelo intacta desde la fundación del estado colonial, la cual, a pesar de las reformas propuestas en los años sesenta y setenta, sigue beneficiando a los grandes propietarios. Según Pino et al. (2018) en Ecuador:

Las UPA ${ }^{1}$ menores a 3 ha son el $52.78 \%$ del total nacional, pero apenas representan el $3.52 \%$ del total de la superficie cultivada del país; mientras que las UPA de más de 50 ha son el $6.41 \%$ del total, pero concentran el $60.72 \%$ del área cultivada a nivel nacional. (p. 10)

Frente a estos problemas, históricamente acumulados, es necesario repensar el futuro del sector agrícola. Tal y como lo indica Naranjo (2016), la transformación agrícola podría orientarse hacia un marco de carácter incluyente que respete y promueva la soberanía alimentaria, la sostenibilidad ambiental, la equidad y el bienestar de los pequeños y medianos productores, sobre todo en el escenario actual, caracterizado por el incremento de la demanda de alimentos, los cambios dramáticos en las condiciones climáticas que afectan su producción y, más recientemente, los efectos de la covid-19.

Según la Organización de las Naciones Unidas para la Alimentación y la Agricultura (FAO, 2020), es urgente y necesario trabajar en medidas tales como incrementar y mejorar la producción agrícola, erradicar la inse- 
guridad alimentaria, racionalizar el consumo de alimentos y la nutrición, mejorar los medios de subsistencia de los productores rurales, garantizar condiciones más justas de intercambio entre productores agrícolas y consumidores, incrementar la resiliencia de los productores agrícolas y reforzar la gobernanza de los sistemas de producción agrícola. A las prioridades señaladas por la FAO, hay que agregar otra forma de desigualdad que caracteriza las relaciones en la economía familiar campesina: la desigualdad de género.

Las condiciones de desigualdad de género son un obstáculo que hay que enfrentar y resolver, pues producen y reproducen una distribución social y sexual del trabajo, donde recae sobre las mujeres las actividades reproductivas no asalariadas del hogar y asigna a los hombres las actividades productivas asalariadas del mercado de trabajo. De acuerdo a datos del Instituto Nacional de Estadística y Censos de Ecuador (INEC), las mujeres ocupan cuatro veces más tiempo que los hombres en el cuidado de menores y adultos en el hogar, lo que limita su ocupación remunerada fuera de él, en un $90 \%$ de los casos, son las mujeres las responsables del trabajo doméstico no remunerado. Esta situación es responsable de que 5 de cada 10 mujeres mayores de 13 años vivan subordinadas a una relación de pareja que las hace dependientes económicamente y víctimas de múltiples formas de violencia; de hecho 8 de cada 10 mujeres han sido víctimas de alguna forma de violencia, sea esta patrimonial, sexual, física o psicológica (VaIle, 2020; Pérez y Agenjo, 2018).
En lo que respecta a la producción agrícola de alimentos, hay que intervenir, por lo menos, en dos direcciones: garantizar la producción sostenible y asegurar su distribución segura, justa y accesible, condiciones que se cumplen dentro de una agricultura sostenible, definida por Sarandón (2020) como un sistema que:

Es capaz de mantener en el tiempo un flujo de bienes y servicios que satisfagan las necesidades alimenticias, socioeconómicas y culturales de la población, dentro de los límites biofísicos que establece el correcto funcionamiento de los sistemas naturales (agroecosistemas) que lo soportan. (p. 38)

Es probable que los que mejor se adapten a este tipo de agricultura sean los pequeños productores de alimentos organizados en la agricultura familiar campesina, sector que en la actualidad se dedica a actividades de subsistencia; sin embargo, asociados a nivel local podrían mejorar su alcance a diferentes escalas. Como señala Meza (2014), una agricultura de este tipo contribuye al desarrollo equilibrado de las comunidades rurales, al mismo tiempo que garantiza la preservación cultural y de la biodiversidad, incrementando la resiliencia comunitaria y la adaptación al cambio climático.

Con el propósito de promover la producción y distribución de alimentos agrícolas con las características anteriores, en 2020 se contactó con una asociación de productoras agrícolas del municipio El Chaco, en la provincia Napo, Ecuador. El objetivo inicial fue fortalecer sus capacidades productivas y 
de comercialización, pues, según manifestaban, sus productos ya tenían acogida entre los consumidores de la ciudad del Tena. Sin embargo, bajo las condiciones impuestas por la covid-19, a partir de abril de 2020 sus ventas bajaron considerablemente. Dentro de los objetivos específicos, se planteó el diseño y la validación participativa de una estrategia de distribución basada en un circuito de proximidad comercial, consistente en anular o bajar la intermediación comercial para incrementar los ingresos de las productoras, lo que iría de la mano de reducir el recorrido de los productos a espacios geográficos próximos para acercar a productores y consumidores de manera humana, con el fin de fortalecer la confianza y el capital social (Ranaboldo y Marta, 2014; Comisión Económica para América Latina y el Caribe [CEPAL], 2013). La aplicación de un circuito de proximidad comercial también propicia la producción y consumo de alimentos sanos, a precios accesibles y justos.

Una vez diseñada la estrategia de distribución, se pretendía su validación en pruebas piloto; sin embargo, en esta fase del proceso se identificaron debilidades organizativas y administrativas de la asociación de productoras que impedían la implementación de forma inmediata de la estrategia. Por un lado, surgieron conflictos de integración entre las socias, pues se percibía que una de las funciones de la asociación era generar empleo y resolver las necesidades económicas de las asociadas. Este fenómeno ha sido descrito como asociacionismo (Batres, 2019) y generalmente va acompañado de un segundo factor que evidencia que la estruc- tura administrativa que se adopta en este tipo de organizaciones no se ajusta a los modelos culturales del grupo y rebasa las capacidades de sus integrantes.

Más adelante, en el análisis de los resultados, se discutirán las características de la estrategia de distribución diseñada para que pueda ser implementada y con el objeto de ofrecerla como modelo para experiencias similares, así como los factores organizativos y administrativos a tener en cuenta que no fueron considerados en esta experiencia.

\section{MATERIALES Y MÉTODOS}

De acuerdo con los datos del Gobierno Autónomo Descentralizado Municipal (GADM) de El Chaco, la población cantonal se estimó en 10236 personas en el año $2020-51 \%$ hombres y $49 \%$ mujeres-, un $35 \%$ de la cual se dedica a actividades agropecuarias, principalmente la crianza de ganado vacuno, porcino y aves de corral. Entre los productos agrícolas destacan el tomate de árbol y la naranjilla, aunque se pueden encontrar otros productos (GADM El Chaco, 2020). Por otro lado, el municipio es reconocido por estar ubicado en una zona rica en recursos hídricos provenientes del Parque Nacional Cayambe-Coca y el Parque Nacional Sumaco-Napo-Galeras, así como de la subcuenca alta del río Coca, que cuenta con 22 proyectos hidroeléctricos en diferentes etapas de desarrollo (Torres et al., 2018). En contraste con lo anterior, el Instituto Nacional de Estadística y Censos de Ecuador (INEC) estima que el $63 \%$ de la población del cantón vive en la pobreza por necesidades bá- 
sicas insatisfechas (INEC, 2016), 40 puntos por arriba del porcentaje nacional, lo cual despertó el interés por trabajar en la zona. El contacto inicial con las productoras de la Asociación Valle de El Chaco: del productor al consumidor ( $\mathrm{AVCh}$ ) se dio en enero de 2020. Después de conversar sobre la idea del proyecto, se pretendía comenzar su ejecución en marzo del mismo año. Sin embargo, con la llegada de la covid-19, se postergó la ejecución, esperando que las condiciones lo permitieran. En el mes de julio se tomó la decisión de reactivar el proyecto, variando la modalidad presencial, definida en un inicio, a una modalidad virtual para la mayoría de las actividades, lo cual permitió avanzar sin comprometer la seguridad de los participantes.

Para diseñar la estrategia de comercialización basada en un circuito de proximidad comercial se recolectaron datos de la oferta y la demanda de productos agrícolas. En el caso de la oferta, se realizaron entrevistas estructuradas a una muestra intencionada de seis productoras de la AVCh, y, para efectos de triangulación de los datos, se realizaron tres observaciones directas en el sitio de distribución de la asociación. En el caso de la demanda, se realizaron entrevistas estructuradas a una muestra intencionada de 17 consumidores potenciales de la ciudad del Tena, basando su integración en tres criterios: que conocieran los productos de la AVCh, que com- praran sus alimentos agrícolas en la ciudad del Tena y que tuvieran interés en el proyecto. El listado inicial de informantes estuvo integrado por ocho personas, y por el mecanismo de Bola de Nieve se llegó a nueve personas más.

En todo este proceso de recolección, organización y análisis de datos participaron siete estudiantes de la Universidad Regional Amazónica Ikiam, el docente investigador a cargo del proyecto y una técnica de la Dirección Provincial del Ministerio de Agricultura, Ganadería, Acuacultura y Pesca de Napo, quien acompaña y asesora a la asociación de productoras. La recolección y organización de datos se realizó con la herramienta Kobotoolbox ${ }^{2}$. Por otra parte, el análisis de la información abarcó dos aspectos: la identificación de los elementos fundamentales de la estrategia de comercialización para su diseño y ejecución piloto, y el análisis $\mathrm{DAFO}^{3}$ de la AVCh.

Una vez concluida la preparación del informe del proyecto, este se entregó a la asociación de productoras y a su asesora para su implementación inicial. En dicha fase, los colaboradores de la Universidad Regional Amazónica Ikiam ofrecieron su acompañamiento para identificar acciones de continuidad del proyecto.

\section{RESULTADOS Y DISCUSIÓN}

Para la presentación y discusión de los hallazgos se procedió, en primer lugar, a ca-

${ }^{2}$ Data Collection Tools for Challenging, software de código abierto y gratuito creado por Kobo Inc., Harvard (HHI) y Brigham and Women's Hospital, en 2020.

${ }^{3}$ Análisis de las debilidades, amenazas, fortalezas y oportunidades. El acrónimo en inglés es SWOT: strengths, weaknesses, opportunities y threats, y se acerca mejor al análisis que se aplicó en este artículo. 
racterizar la oferta de productos agrícolas brindados por la AVCh, para luego contrastar esta información con la demanda de los consumidores potenciales que se entrevistaron. Con la información de las dos secciones anteriores se integró la estrategia de distribución basada en un circuito de proximidad comercial que se presenta y discute en el tercer subtítulo. Finalmente, se efectuó un análisis de las condiciones organizativas y administrativas que debería considerar la AVCh para implementar la estrategia en ciernes; dicho análisis se presenta en el cuarto subtítulo de este artículo.

\section{Caracterización general de la oferta de la Asociación Valle de El Chaco: del productor al consumidor}

La AVCh se constituyó legalmente como asociación en junio de 2018, mediante el Acuerdo Ministerial N. ${ }^{\circ}$ 024-DPANAPO-2017. En un inicio, contaba con 23 integrantes que se propusieron comercializar sus productos en tres puntos de venta de la provincia de Napo, ellos son Baeza, El Chaco y la ciudad de Tena. De acuerdo con la información proporcionada por las socias, en el año 2020 su oferta estaba integrada por 43 productos agropecuarios y 11 productos elaborados, como quesos, manjar, chifles, tostados, etc.

Con la información proporcionada por las productoras, se estableció el tiempo de manejo poscosecha o elaboración de sus productos $\left(T_{M E}\right)$, que incluye: el tiempo aproximado en horas usado en la cosecha o elaboración $\left(T_{1}\right)$, las horas de alma- cenamiento $\left(T_{2}\right)$ y las horas usadas en el transporte hasta el lugar de venta $\left(T_{3}\right)$. En todos los casos, $T_{3}$ equivale a tres horas desde El Chaco hasta la ciudad del Tena, con una distancia de 100 kilómetros por la ruta más transitada. En cuanto a TME varía dentro del conjunto de productos en un rango de 5 a 24 horas. Entre los productos agropecuarios con TME cercanos a un día (18 a 24 horas) se encuentran aquellos con alguna complejidad en la cosecha o almacenamiento, como el fréjol, el tomate de árbol, la papa china, etc. El grupo de productos cuyo Tme es cercano a medio día (10 a 17 horas) incluye productos como la acelga, el brócoli y los quesos. Finalmente, los productos con un rango de Tme más corto (5 a 9 horas) son el tomate cherry, el zapallo, el maqueño, las hierbitas y la lechuga de hoja. En síntesis, el TME promedio del conjunto de productos ofrecidos por la AVCh es de 17 horas.

Para analizar el volumen de producción o cantidad de unidades disponibles para la venta por semana (Vpus) se usaron las diferentes medidas de venta descritas durante las entrevistas realizadas a productoras. Por ejemplo, peso en gramos para productos como queso, tostones y chifles; peso en libras para productos como tomate, zanahoria y naranjilla; y atado para hierbas, acelga y apio. El Vpus está definido por la experiencia de ventas de las productoras, más que por el uso de la capacidad de producción (Cpus) o cantidad máxima de unidades que en las condiciones actuales se podría producir por semana. Sería útil contar con información adicional para es- 
tablecer la Cpus y comparar con la Vpus, con la finalidad de conocer su diferencial y, a partir de esto, valorar la posibilidad de escalar el modelo de negocio, si bien esto rebasa el alcance de este artículo. Lo que puede hacerse con la información disponi- ble es comparar la VPUS con las unidades vendidas por semana (Uvs) y estimar en esa diferencia el porcentaje de pérdida o ganancia en ventas de la AVCh. Estos y otros datos pueden deducirse de la tabla 1.

Tabla 1. Caracterización de la oferta de productos agropecuarios de la AVCh

\begin{tabular}{|c|c|c|c|c|c|c|c|c|}
\hline Producto & $\mathrm{T}_{1}$ & $\mathbf{T}_{2}$ & $\begin{array}{c}T_{M E} \\
\left(T_{1}+T_{2}+3\right)\end{array}$ & $\mathrm{V}_{\text {PUS }}$ & $\mathbf{U}_{\mathrm{vs}}$ & PVP & $\begin{array}{c}\text { Ventas } \\
\left(\mathrm{U}_{\mathrm{vs}} \times \mathrm{PVP}\right)\end{array}$ & $\begin{array}{c}\text { Diferencial } \\
\left(\mathrm{V}_{\text {Pus }}-\mathrm{U}_{\mathrm{VS}}\right) \text { ) } \\
\text { PVP } \\
\end{array}$ \\
\hline $\begin{array}{l}\text { Hojas (acelga, perejil, } \\
\text { apio, etc.) }\end{array}$ & 1 & 13 & 17 & 5 & 4 & $\$ 0.25$ & $\$ 1.00$ & $\$ 0.25$ \\
\hline Babaquillo & 1 & 15 & 19 & 25 & 14 & $\$ 1.00$ & $\$ 14.00$ & $\$-11.00$ \\
\hline Chocho & 3 & 15 & 21 & 26 & 24 & $\$ 1.00$ & $\$ 24.00$ & $\$-2.00$ \\
\hline Col & 2 & 14 & 19 & 40 & 31 & $\$ 1.00$ & $\$ 31.00$ & $\$-9.00$ \\
\hline Fréjol & 4 & 17 & 24 & 25 & 24 & $\$ 1.00$ & $\$ 24.00$ & $\$-1.00$ \\
\hline Hierbita & 1 & 1 & 5 & 15 & 15 & $\$ 0.25$ & $\$ 3.75$ & \\
\hline Limones & 1 & 15 & 19 & 4 & 4 & $\$ 1.00$ & $\$ 4.00$ & \\
\hline Manjar de leche & 4 & 12 & 19 & 40 & 26 & $\$ 1.00$ & $\$ 26.00$ & $\$-14.00$ \\
\hline Mermelada & 3 & 12 & 18 & 12 & 8 & $\$ 1.00$ & $\$ 8.00$ & $\$-4.00$ \\
\hline Naranjilla & 1 & 15 & 19 & 10 & 10 & $\$ 1.00$ & $\$ 10.00$ & \\
\hline Papa china & 1 & 15 & 19 & 9 & 8 & $\$ 1.00$ & $\$ 8.00$ & $\$-1.00$ \\
\hline Pepino dulce & 2 & 18 & 23 & 20 & 15 & $\$ 1.00$ & $\$ 15.00$ & $\$-5.00$ \\
\hline Queso de mesa & 4 & 3 & 10 & 100 & 60 & $\$ 2.00$ & $\$ 120.00$ & $\$-80.00$ \\
\hline Queso mozarela & 4 & 8 & 15 & 200 & 190 & $\$ 3.00$ & $\$ 570.00$ & $\$-30.00$ \\
\hline Tomate cherry & 1 & 5 & 9 & 10 & 8 & $\$ 1.00$ & $\$ 8.00$ & $\$-2.00$ \\
\hline Tomate de árbol & 4 & 18 & 25 & 65 & 51 & $\$ 1.00$ & $\$ 51.00$ & $\$-14.00$ \\
\hline Tomate de riñón & 3 & 18 & 24 & 50 & 44 & $\$ 1.00$ & $\$ 44.00$ & $\$-6.00$ \\
\hline Zapallo & 3 & 3 & 9 & 12 & 8 & $\$ 1.25$ & $\$ 10.00$ & $\$-5.00$ \\
\hline Zanahoria Blanca & 3 & 18 & 24 & 10 & 10 & $\$ 1.00$ & $\$ 10.00$ & \\
\hline Banana & 2 & 16 & 21 & 10 & 10 & $\$ 1.00$ & $\$ 10.00$ & \\
\hline Pimiento & 2 & 12 & 17 & 12 & 11 & $\$ 1.00$ & $\$ 11.00$ & $\$-1.00$ \\
\hline Brócoli & 2 & 12 & 17 & 8 & 8 & $\$ 1.20$ & $\$ 9.60$ & \\
\hline Mora & 4 & 8 & 15 & 10 & 10 & $\$ 1.00$ & $\$ 10.00$ & \\
\hline Cebolla & 4 & 16 & 23 & 8 & 8 & $\$ 1.00$ & $\$ 8.00$ & \\
\hline Hongos & 2 & 6 & 11 & 8 & 8 & $\$ 2.50$ & $\$ 20.00$ & \\
\hline $\begin{array}{l}\text { Frutas de temporada } \\
\text { (papaya, naranja, } \\
\text { maracuyá, guayaba, etc.) }\end{array}$ & 3 & 12 & 18 & 10 & 10 & $\$ 1.00$ & $\$ 10.00$ & \\
\hline Achocha & 2 & 8 & 13 & 5 & 5 & $\$ 1.00$ & $\$ 5.00$ & \\
\hline Ají & 2 & 6 & 11 & 15 & 15 & $\$ 0.5$ & $\$ 7.5$ & \\
\hline
\end{tabular}

Nota: PVP: precio de venta por producto.

Fuente: Elaborada por el autor a partir de entrevistas a productoras de la AVCh (2020). 
El monto de ventas genera un ingreso semanal de $\$ 1071.85$, mientras que la suma de diferenciales se estima en \$185; esto representa un porcentaje de pérdida del $17 \%$ si se acepta que esos productos no se pondrán a la venta nuevamente. Dentro del conjunto de productos se distingue el queso mozarela, que representa el 58 \% del total de ventas y el $16 \%$ de las pérdidas; por su comportamiento, podría identificarse como el producto con mayor participación dentro de la oferta de la AVCh. El segundo producto que destaca es el queso de mesa, que produce un $12 \%$ del ingreso por ventas y un $43 \%$ de pérdida; este resultado cuestiona la pertinencia de incluir este producto dentro de la oferta de la AVCh. Este mismo comportamiento también se observa en productos elaborados como el manjar de leche y las mermeladas, mientras que, indicadores positivos se observan en productos como el fréjol y el tomate riñón, aunque con valores cuantitativamente más bajos.

Otro factor que incide directamente en la oferta de la AVCh es la disponibilidad continua de los productos ofrecidos por algunas socias. La oferta colectiva de la asociación depende del trabajo organizado y responsable de todo el grupo; cualquier situación que influya en estos aspectos puede afectar, tarde o temprano, la oferta de productos. Este tema se abordará con mayor detalle más adelante, cuando se analicen las condiciones organizativas y administrativas que deberían lograrse para implementar la estrategia de comercialización sugerida.

El proceso de cosecha, almacenamiento y transporte de productos agropecuarios fue descrito por las productoras de la siguiente manera:

a. La cosecha es una actividad familiar que se hace por la mañana con herramientas básicas y equipo de protección como gorro, guantes, mascarilla y botas de caucho.

b. Los productos cosechados se llevan a las casas de las productoras, donde se lavan con agua y se depositan en bandejas para ser trasladados en carretilla o transporte urbano hasta el centro de acopio ubicado en el Mercado de El Chaco.

c. Una vez allí, los productos se limpian y empacan siguiendo medidas de bioseguridad como la limpieza del piso con agua caliente; la desinfección de mesas con paños untados en alcohol, y el uso de mascarilla, overol, gorro, guantes y botas de caucho.

d. Finalmente, los productos son trasladados a su lugar de venta empacados en fundas plásticas o tarrinas usando el transporte público.

El lugar de distribución en la ciudad de Tena es un espacio en el parqueo de las oficinas del Ministerio de Agricultura, Ganadería, Acuacultura y Pesca, en el cual se colocan dos carpas plásticas para proteger de la lluvia y el sol los productos exhibidos en las mesas. En el lugar se tiene a disposición un servicio sanitario; en el momento de la compra, se utiliza mascarilla, mandril y guantes, y se desinfectan las manos y el dinero con alcohol. En general, se observa que las productoras de la AVCh están familiarizadas 
con las medidas de bioseguridad sugeridas por los consumidores, por lo cual sería posible protocolizarlas en todo el manejo poscosecha.

Características generales de la demanda de los consumidores potenciales de los productos agropecuarios de la AVCh

Para realizar la caracterización que sigue se entrevistó a 17 personas de ellas cinco fueron hombres y 12 mujeres. La edad promedio del grupo es de 40 años, en un rango entre 28 y 60 años. Todas las personas entrevistadas viven en la ciudad de Tena, compran sus productos en esa ciudad, conocen los productos ofrecidos por la AVCh y manifestaron su disposición para apoyar cual- quier iniciativa que los haga más accesibles comercialmente.

Se indagó sobre los productos que las personas entrevistadas consumen semanalmente, obteniendo un listado de 54 productos agropecuarios, de los cuales 22 están incluidos en la oferta de la AVCh. Para su comparación, los productos demandados y ofrecidos se clasificaron en tres columnas según su frecuencia de demanda dentro del conjunto de productos comprados semanalmente. De los 22 productos ofrecidos por la AVCh, ocho se ubican dentro de los productos más demandados por los consumidores potenciales, 10 dentro de la demanda media y seis dentro de la demanda baja.

Tabla 2. Listado de productos demandados por las personas entrevistadas

\begin{tabular}{|c|c|c|c|c|c|}
\hline $\begin{array}{l}\text { Productos con } \\
\text { mayor demanda }\end{array}$ & (f) & $\begin{array}{c}\text { Productos con } \\
\text { demanda media }\end{array}$ & (f) & $\begin{array}{c}\text { Productos con menor } \\
\text { demanda }\end{array}$ & (f) \\
\hline Tomate & 12 & Choclo & 5 & Achocha & 2 \\
\hline Рapa & 11 & Col & 5 & Fréjol & 2 \\
\hline Cebolla colorada & 11 & Manzanas & 5 & Hierbita & 2 \\
\hline Pimiento & 11 & Papaya & 5 & Limón & 2 \\
\hline Acelga & 10 & Aguacate & 4 & Miel de Abeja & 2 \\
\hline Plátano verde & 10 & Cebolla blanca & 4 & Palmito & 2 \\
\hline Fresa & 9 & Culantro & 4 & Plátano maduro & 2 \\
\hline Zanahoria & 9 & Hongos & 4 & Sandía & 2 \\
\hline Banana & 8 & Maracuyá & 4 & Zapallo & 2 \\
\hline Mandarinas & 8 & Perejil & 4 & Nabo & 2 \\
\hline Yuca & 8 & Rábano & 4 & Papa nabo & 1 \\
\hline Brócoli & 7 & Remolacha & 4 & Garabato yuyo & 1 \\
\hline Coliflor & 7 & Suquini & 4 & Guayaba & 1 \\
\hline Espinaca & 7 & Tomate de árbol & 4 & Puerro & 1 \\
\hline Mora & 7 & Uvas & 4 & Vainitas & 1 \\
\hline Ajo & 6 & Albahaca & 3 & & \\
\hline Habas & 6 & Apio & 3 & & \\
\hline \multirow[t]{4}{*}{ Pepino } & 6 & Chochos & 3 & & \\
\hline & & Espárrago & 3 & & \\
\hline & & Lechuga & 3 & & \\
\hline & & Melón & 3 & & \\
\hline
\end{tabular}

Nota: En negrita, los productos ofrecidos por la AVCh y demandados por las personas entrevistadas; f: frecuencia.

Fuente: Elaborada por el autor a partir de entrevistas a consumidores de la ciudad de Tena. 
En las tablas 1 y 2 se pueden identificar las correspondencias entre los productos ofrecidos por la AVCh y los productos que consumen semanalmente las personas entrevistadas. Los 22 productos que aparecen en ambas tablas constituyen la base sobre la cual debería definirse la estrategia de comercialización. De cualquier forma, las productoras podrían mejorar su oferta incorporando parte de los productos que demandan los consumidores y que aún no ofrecen, así como promocionando productos con los que cuentan y que no son consumidos por las personas entrevistadas. Una vez que se consideren e integren esas posibilidades, se pueden atender otras cuestiones que están vinculadas a las expectativas de los consumidores potenciales y que deben ser tenidas en cuenta; a continuación, se revisan algunas de ellas.

Con respecto a los sitios en donde usualmente compran los consumidores potenciales, a través de las entrevistas pudieron establecerse los elementos que les agradan y disgustan de esos lugares. En orden de aparición se destacan los siguientes:

a. Que se vendan productos orgánicos y frescos.

b. Que no se despache en fundas plásticas o tarrinas.

c. Cercanía con el productor: amabilidad en la venta, conocer a quién produce y reducir la intermediación.

d. Que tengan un precio económico y asequible. e. Que en el lugar de venta se sigan medidas de bioseguridad tales como distanciamiento entre personas y limpieza de los productos, como mínimo.

f. Variedad y continuidad en los productos ofrecidos, de preferencia locales.

g. Estética del lugar: orden y limpieza, sobre todo.

A las medidas de bioseguridad ya señaladas, los consumidores potenciales agregaron las siguientes: el uso de mascarilla durante la transacción; la desinfección continua de productos y dinero con alcohol; que el espacio de venta sea ventilado y amplio; el uso de bandejas de desinfección para calzado, y el uso de guantes en la manipulación de los productos.

\section{Identificación y descripción de la estrate- gia de comercialización de productos basa- da en un circuito de proximidad comercial}

Para lograr que el sistema de compra-venta de productos se acerque al concepto de los circuitos de proximidad comercial, se identificó la opción de ofrecer y entregar canastas de productos agropecuarios directamente al consumidor. Esta opción simplifica la cadena de distribución y reduce los intermediarios. En adelante, este sistema de compra-venta será nombrado ashanga de alimentos, palabra que en idioma kichwa se usa para referirse a una canasta artesanal en la que se éstos se recolectan.

Entre los entrevistados se indagó si conocían el sistema de compra de productos a pedido y entrega en una canasta o ashanga de alimentos, lo que arrojó que todos conocen esta modalidad de compra-venta. Dentro 
de las opciones identificadas, se eligieron en orden las siguientes: entrega en su casa, pagando un importe adicional de transporte (A); entrega en un lugar cercano a su casa, un día y horario específico (B); entrega en un lugar único de Tena, en un día y horario definido (C).

El promedio de disposición de pago adicional por transporte de la ashanga de alimentos para las opciones que lo requieren ( $\mathrm{A} y$ B) fue de \$1.58; cantidad que es mayor al costo real de un servicio de traslado en taxi en la ciudad de Tena (\$1.25). En el caso de la opción que no conlleva pago adicional de transporte $(\mathrm{C})$, se debe agregar que implica menos complejidades logísticas, pues las productoras ya cuentan con un centro de distribución ubicado en el centro de la ciudad de Tena.

Con los datos disponibles se logró establecer el monto semanal promedio de compra de los clientes regulares de la AVCh, así como el monto semanal promedio de compras de los consumidores entrevistados. Los clientes actuales de las productoras realizan una compra semanal de $\$ 15.00$, mientras que los consumidores entrevistados realizan una compra promedio de $\$ 21.69$. Si al promedio de compra de las personas entrevistadas se agrega el costo real de distribución (\$1.25) y se elige la primera opción ( $A)$, los consumidores entrevistados deberían pagar $\$ 23.00$ por una ashanga de alimentos entregada en la puerta de su domicilio; en el caso de los clientes actuales de la AVCh, estos deberían pagar $\$ 16.25$ por el mismo concepto. La opción B solo varía en el lugar de entrega, un sitio cercano al domicilio del comprador.

Para acomodar la estrategia de distribución a las condiciones generales de los consumidores potenciales, en el marco de la emergencia provocada por la covid-19, se averiguó el día y horario en que se preferiría recibir la ashanga de alimentos. Las respuestas obtenidas no reflejan una preferencia específica, por lo tanto, se asume que en condiciones de confinamiento cualquier día de la semana y horario podrían funcionar para la entrega, siempre y cuando se haga el pedido con una anticipación mínima de 12 horas a través de medios como WhatsApp.

\section{Análisis de las condiciones organizativas y administrativas que debería tomar en cuenta la AVCh para implementar la estra- tegia de distribución de productos}

Para el análisis de las condiciones organizativas y administrativas se usó la herramienta DAFO (debilidades, amenazas, fortalezas y oportunidades), cuyo resultado se discute a continuación.

Las debilidades son aspectos internos de la AVCh que afectan la implementación de cualquiera de las opciones de la estrategia de comercialización de sus productos agropecuarios. Las debilidades identificadas fueron las siguientes:

a. La oferta actual de productos agropecuarios de la AVCh coincide en un 40\% con los productos demandados por los consumidores potenciales, cinco de ellos corresponden a una demanda baja.

b. El $29 \%$ de los productos agropecuarios ofertados por la AVCh podría estar produciendo una pérdida en ventas semanales del $17 \%$.

c. Dentro de la oferta de productos agro- 
pecuarios de la AVCh, los elaborados son los que mayores ventas semanales generan, pero con mayor riesgo de pérdida.

d. La disponibilidad continua de la oferta de productos de la AVCh es afectada por conflictos internos entre las socias.

e. Las socias de la AVCh no cuentan con recursos tecnológicos apropiados y la capacitación para atender los pedidos realizados por WhatsApp.

f. No se llevan registros contables y administrativos de la asociación para identificar el funcionamiento de su modelo de negocio.

Las amenazas son condiciones de origen externo que afectan la venta actual de productos agropecuarios de la AVCh y en el futuro podrían afectar la implementación de la estrategia de comercialización basada en los circuitos de proximidad comercial. En el análisis realizado se identificaron las siguientes amenazas:

a. La vía principal entre El Chaco y la ciudad de Tena es vulnerable a derrumbes, aludes y socavamientos continuos que cortan el tránsito vehicular.

b. El surgimiento de nuevas variantes de la covid-19, de las cuales no se conoce lo suficiente para estimar las medidas de bioseguridad necesarias.

c. La percepción cultural del asociacionismo impide que la AVCh haga eficiente el modelo de negocio de su emprendimiento.

d. Su contexto de referencia, como acceso a formación, créditos y tierra para cultivo, limita sus oportunidades productivas. e. La contracción de las instituciones públicas dedicadas a apoyar la economía popular y solidaria incrementa el abandono estatal de este sector.

Las debilidades $d$, e y $f$, y las amenazas $c, d$ y e giran alrededor de un eje común, el asociacionismo, consistente en el surgimiento de numerosas asociaciones promovidas por instituciones públicas con estructuras de funcionamiento administrativo predefinidas, altamente burocratizadas y que no responden a los modelos de organización y participación cultural de las comunidades en donde se constituyen. Diversas instituciones públicas ofrecen su apoyo a los grupos comunitarios solo si están constituidos en asociaciones legalmente reconocidas; esta posibilidad precipita la búsqueda de la institucionalización de los grupos comunitarios sin acumular las capacidades de gestión que conlleva la economía popular y solidaria. Una vez registradas en una institución pública, las asociadas demandan de sus dirigentes la canalización de fondos públicos y el crecimiento de la productividad del grupo; si, eventualmente, se tuviera la atención de organismos externos, se incrementaría la conflictividad del grupo, pues se provocarían dudas en el manejo de los recursos y en su distribución. Este fenómeno ha sido observado en tres asociaciones productivas que se han acompañado con anterioridad en la Amazonía ecuatoriana (Batres, 2019; Bacquet y Batres, 2018).

Por su parte, las debilidades $a, b$ y $c$ se relacionan con la definición de la oferta de productos agropecuarios de la AVCh y, en conjunto con las fortalezas y oportunida- 
des que se verán más adelante, apuntan a una redefinición de la misma. Las amenazas a y b son particulares donde la primera no está dentro del margen de maniobra de las productoras, mientras que para enfrentar la segunda sería necesario incrementar y protocolizar las medidas de bioseguridad en el manejo poscosecha y en la venta de los productos, para lo cual hay experiencia y disposición por parte de las productoras.

En cuanto a las fortalezas o capacidades internas acumuladas por la AVCh, se mencionaron las siguientes:

a. La oferta de productos se ajusta a las características deseadas en los circuitos de proximidad comercial y provienen de una distancia no mayor a 100 kilómetros, con un tiempo promedio de entrega de 17 horas desde su cosecha.

b.Son, por lo tanto, alimentos frescos y saludables producidos para los consumidores por mujeres dentro de la agricultura familiar campesina.

c. En su distribución no participan intermediarios, por lo cual se puede maximizar la ganancia en ventas y las productoras podrían recibir un pago justo por su trabajo.

d. Los precios de sus productos son accesibles y están por debajo de lo que actualmente pagan o están dispuestos a pagar los consumidores potenciales.

Finalmente, se identificaron las oportunidades, que corresponden a las condiciones del entorno que pueden ser aprovechadas por la AVCh para mejorar su negocio productivo. En el análisis se nombraron las siguientes oportunidades: a. Los consumidores potenciales conocen y valoran las opciones de distribución de las ashangas de alimentos.

b. En las condiciones sanitarias impuestas por la covid-19, las estrategias de distribución identificadas reducen los riesgos de contagio una vez que se adoptan las medidas de bioseguridad necesarias.

c. Las estrategias de distribución definidas pueden reducir el tiempo usado por los consumidores para la compra de sus productos agropecuarios, a la vez que disminuyen el tiempo y esfuerzo invertidos por las productoras para vender sus productos.

d. La implementación de las estrategias identificadas elimina la intermediación de terceros entre productores y consumidores, lo que significa una reducción en el precio de venta por producto y un incremento en las ganancias percibidas por las productoras de la AVCh.

Identificados los componentes del análisis DAFO, se pueden cruzar entre sí para identificar estrategias de acción; por ejemplo, cruzando las capacidades internas con las oportunidades del contexto, se pueden definir estrategias de crecimiento; mientras que, relacionando fortalezas con amenazas, se pueden definir acciones reactivas para contrarrestar las condiciones negativas del entorno. En la relación entre debilidades y oportunidades, pueden definirse estrategias adaptativas para ajustar la actividad productiva al contexto. Por último, examinando las debilidades y las amenazas, se pueden definir estrategias de sobrevivencia que au- 
menten la capacidad interna para reducir el impacto de las amenazas externas. En la tabla 3 se resumen las estrategias que deberían im- plementarse de manera preliminar o en conjunto con las tres opciones de distribución de productos antes identificadas.

Tabla 3. Estrategias de aplicación inmediata identificadas en el análisis DAFO, caso AVCh

\begin{tabular}{|c|c|c|c|}
\hline $\begin{array}{l}\text { Estrategias de } \\
\text { crecimiento } \\
\text { (fortalezas }+ \\
\text { oportunidades) }\end{array}$ & $\begin{array}{c}\text { Estrategias } \\
\text { reactivas } \\
\text { (fortalezas }+ \text { amenazas })\end{array}$ & $\begin{array}{c}\text { Estrategias adaptativas } \\
\text { (debilidades }+ \\
\text { oportunidades) }\end{array}$ & $\begin{array}{c}\text { Estrategias de } \\
\text { sobrevivencia } \\
\text { (debilidades }+ \\
\text { amenazas) }\end{array}$ \\
\hline $\begin{array}{l}\text { Implementar las tres } \\
\text { opciones de } \\
\text { distribución de } \\
\text { ashangas de } \\
\text { alimentos. }\end{array}$ & $\begin{array}{l}\text { Promocionar los valores } \\
\text { agregados de la AVCh en } \\
\text { el contexto provocado } \\
\text { por la covid-19 de } \\
\text { productos próximos, } \\
\text { saludables y frescos } \\
\text { producidos por mujeres a } \\
\text { precios accesibles. }\end{array}$ & $\begin{array}{l}\text { Ampliar la oferta de } \\
\text { productos de la AVCh, } \\
\text { integrando aquellos } \\
\text { que consumen los } \\
\text { demandantes y que en } \\
\text { el presente no se } \\
\text { ofrecen. }\end{array}$ & $\begin{array}{l}\text { Capacitar a las socias } \\
\text { de la AVCh en medidas } \\
\text { de bioseguridad, } \\
\text { administración y uso de } \\
\text { TIC. Resolver conflictos } \\
\text { internos, adoptando los } \\
\text { principios definidos en } \\
\text { el artículo } 4 \text { de la Ley } \\
\text { Orgánica de la } \\
\text { Economía Popular y } \\
\text { Solidaria del Ecuador } \\
\text { (LOEPS, 2011). Ajustar } \\
\text { el modelo asociativo a } \\
\text { los artículos } 15 \text { y } 16 \text { de } \\
\text { la LOEPS }\end{array}$ \\
\hline
\end{tabular}

Fuente: Elaborada por el autor a partir del análisis de los resultados de entrevistas, la observación directa y la discusión participativa de resultados.

\section{CONCLUSIONES}

La agricultura familiar campesina es una de las principales fuentes de producción de alimentos agropecuarios en Ecuador. Sin embargo, su actividad productiva se ve amenazada por factores como el despoblamiento rural, el modelo agrícola excluyente que caracteriza al país y la contracción de la inversión pública. Estas condiciones se ven agravadas por el contexto de emergencia sanitaria provocada por la covid-19 a partir de abril de 2020, de ahí que se prevé que los efectos de la pandemia profundizarán aún más la crisis que vive este sector productivo. Es por ello que se hace necesario desarrollar acciones paliativas que reviertan los riesgos que esto produce para la soberanía y seguridad alimentarias de Ecuador.

En este escenario, se analizó la oferta de productos agropecuarios ofrecidos por una asociación productiva, integrada en su mayoría por mujeres, para evaluar la factibilidad de mejorar su sistema de comercialización a través de opciones basadas en un circuito

${ }^{4}$ La Ley Orgánica de Economía Popular y Solidaria del Ecuador, en sus artículos 15 y 16, acepta que las asociaciones adopten la forma de gobierno interno que mejor se cultura. 
de proximidad comercial. La comparación general de la oferta y la demanda demuestra que es viable implementar este cambio usando tres modalidades de distribución de ashangas de alimentos.

No obstante, antes de hacer operativas las medidas anteriores, es necesario realizar un conjunto de ajustes estratégicos al modelo de negocio. Estos ajustes estratégicos fueron identificados durante la realización del análisis DAFO, y su aplicación permitirá potenciar las fortalezas internas de la asociación, aprovechar las oportunidades del contexto, reducir el efecto de las amenazas externas y superar las debilidades internas.

Por último, los resultados obtenidos corresponden a una fase de investigación del modelo de circuito de proximidad comercial, por lo que no han sido evaluados en funcionamiento. Corresponde a la AVCh y a la asesora del Ministerio de Agricultura, Ganadería, Acuacultura y Pesca su implementación, para lo cual se entregó el informe respectivo y se manifestó la disposición de acompañar y asesorar dicha fase.

\section{REFERENCIAS BIBLIOGRÁFICAS}

Bacquet, C. N. O. y Batres, J. A. (2018). Tejido y educación ambiental en comunidades kichwas de Tena, Ecuador. Revista Rupturas, 9(1), 1-17. https://doi.org/10.22458/rr.v9i1.2226

Batres, J. A. (2019). Emprendimiento sostenible con guadúa en comunidades kichwas de Tena. Revista de Investigación Enlace Universitario, 18(1), 73-86. https://doi.org/10.33789/en$\underline{\text { lace. } 18.48}$

Comisión Económica para América Latina y el Caribe. (2013). Agricultura familiar y circuitos cortos: Nuevos esquemas de producción, comercialización y nutrición. Memoria del seminario sobre circuitos cortos realizado el 2 y 3 de septiembre de 2013. https://bit.ly/2ZzJZtn

Gobierno Municipal Descentralizado de El Chaco. (2020). Implementación de fincas agroecológicas turísticas como estrategia de adaptación al cambio climático. Dirección de Planificación de Proyectos Gobierno Municipal Descentralizado El Chaco.

Instituto Nacional de Estadística y Censos. (2016). Reporte de pobreza por consumo Ecuador 20062014. Instituto Nacional de Estadística y Censos y Banco Mundial. https://bit.ly/317S2hL

Ley Orgánica de Economía Popular y Solidaria. (2011, 10 de mayo). Asamblea Nacional. Registro Oficial 444 de 10 de mayo 2011. https://bit.ly/3vJOSvy

Meza, L. (2014). La agricultura familiar y el cambio climático. En S. Salcedo y L. Guzmán (Ed.), Agricultura familiar en América Latina y el Caribe: Recomendaciones de Política. (pp. 79-100). Organización de las Naciones Unidas para la Alimentación y la Agricultura.https://bit.ly/3pAsP9L 
Naranjo, M. (Coord.). (2016). La política agropecuaria ecuatoriana: hacia el desarrollo territorial rural sostenible: 2015-2025 (Parte I). Ministerio de Agricultura, Ganadería, Acuacultura y Pesca. http://extwprlegs1.fao.org/docs/pdf/ecu183434.pdf

Organización de las Naciones Unidas para la Alimentación y la Agricultura. (2020). Panorama de la inseguridad alimentaria en América Latina y el Caribe. Fondo Internacional de Desarrollo Agrícola (FIDA), Organización Panamericana de la Salud (OPS), Programa Mundial de Alimentos (WFP) y Fondo de las Naciones Unidas para la Infancia (UNICEF). http://www.fao. org/3/cb2242es/cb2242es.pdf

Pino, S. L., Aguilar, H. R., Apolo, A. G. E. y Sislema, L. A. (2018). Aporte del sector agropecuario a la economía del Ecuador. Análisis crítico de su evolución en el período de dolarización. Años 2000-2016. Revista Espacios, 39(32), 7-18. https://bit.ly/3meUoDh

Pérez, A. y Agenjo, A. (2018). Economía feminista: viva, abierta y subversiva. En E. Armendáriz (Ed.). Economía feminista: visibilizar lo invisible. (pp. 6-10). Economistas sin Fronteras. https://bit.ly/3vLwzGt

Ranaboldo, C. y Arosio, M. (2014). Rural-Urban Linkages: Short food chains and local food systems. Working Paper Series N. ${ }^{\circ}$ 129. Latin American Center for Rural Development. https://bit.ly/3jGW6f5

Sarandón, S. J. (2020). El papel de la agricultura en la transformación social y ecológica de América Latina. Cuadernos de la Transformación, N. 11. Friedrich-Ebert-Stiftung. https://bit.ly/3GyDdoi

Srinivasan, S. V. y Rodríguez, A. G. (2016). Pobreza y desigualdades rurales. Perspectivas de género, juventud y mercado de trabajo. Comisión Económica para América Latina y el Caribe. https://bit.ly/2XUTZwH

Torres, M. C., Fierro, V., Páez, S., Plúa, F., Carrera, M. I., Moscoso, N., Cazco, R., Tamayo, G., Ruiz, M., Narváez, M., López, C. E. y Espine, D. (2018). Resultados de las modelaciones biofísicas, valoración económica y propuesta política. Cuenca del río Coca. Escuela Politécnica Nacional y Programa de Naciones Unidas para el Medio Ambiente. https://bit.ly/3pMtKUH

Valle, C. (2020). Atlas de género de Ecuador. Instituto Nacional de Estadística y Censos. https://bit.ly/3bmZKGp

Villacís, B. y Carrillo, D. (2012). País atrevido: la nueva cara sociodemográfica del Ecuador. Edición especial Revista Analítika. Instituto Nacional de Estadística y Censos. https://bit.ly/3BtAXei 